\title{
Sustainability and spinal cord injury: attending to climate change and disability
}

\author{
Marcalee Alexander ${ }^{1}$
}

Received: 7 October 2019 / Accepted: 7 October 2019

(c) International Spinal Cord Society 2019

In July 2019, the UN acknowledged the issues of people with disabilities with respect to climate change as a human rights concern. Of all the causes of disability, spinal cord injury (SCI) with the potential for poikilothermia, difficulties with mobility, sensory dysfunction, and need for external devices easily tops the list as being the most significant condition causing vulnerability to the impacts of climate change. Rising and rapidly changing temperatures, increased flooding, hurricanes, and potential fires could all wreak havoc on our clients with SCIs. Hence, it is imperative that discussions of climate change and disability include the unique issues of persons with SCIs. Likewise, it is important that we, as professionals, look into the nuances of how our clinical practices can be fine-tuned to ascertain that they are sustainable and that we are doing our best to insure people with SCIs maintain their quality of life despite the impacts of climate change.

Societies such as the International Spinal Cord Society are important to ensure appropriately trained professionals care for those with SCIs and educate others about the unique needs of people with SCIs. Globally, we must also ascertain that we consider the needs of people in countries in various phases of development. However, in a culture of diminishing resources this is difficult, and another reason to think about sustainability.

Sustainability is a relatively new but important topic in the professional literature related to people with SCIs. One way of encouraging sustainability is through virtual means to provide health care. In the piece by Tsai et al. [1], the current editor's choice, "The feasibility and usability of a mobile application for performing home evaluations," authors utilized an app to have consumers outline a floor plan of their home in lieu of a home visit so clinicians could

Marcalee Alexander

spinalcordmd@live.com

1 Telerehabilitation International, Birmingham, Alabama, USA appropriately order durable medical equipment. The instructions for using the app were improved during the course of the study and with the improved education, 20 floor plans that were created by consumers were considered useful by clinicians $75 \%$ of the time. Consider the benefits. Elimination of the need for a clinician to physically travel to a person's home, which could be hundreds of kilometers away and take a day of travel would save time of the clinician, decrease cost and lower carbon emissions into the atmosphere. Moreover, as face to face home visits may already have been reduced or even eliminated in many environments, the replacement of this service is important and ensures that the client with SCI is receiving appropriate equipment and that recommendations for their home setup can be provided by clinicians. There is also the paper by Tyagi et al. [2], "Improving quality of life after spinal cord injury in India with telehealth," that documents how two people with SCIs who lived 142 and $1083 \mathrm{~km}$ from the spine center were able to increase their functional status after an initial post discharge decline through the development of a specific home care program and the use of WhatsApp, video chats, and phone calls for administration. In this situation, the individual is receiving a specialized service and there is a similar tremendous saving in miles traveled and resultant decrease in cost and potential carbon emissions.

Virtual health care is an important issue to ponder regarding sustainability; however, there are other concerns. Another facet is the use of electronic devices that could potentially be replaced in nonelectronic format. For instance, is it always necessary to prescribe all the electronic mattresses or beds that people receive? Do we always need to use inexsufflators versus assisted coughing? How many of these devices are unnecessarily prescribed? And when we use these devices, what about the disposable supplies such as tubing that accompany them-where does this go?

Similar to the supplies associated with electronic devices, it is important to consider "throwaways" such as catheters. While disposable catheters may be preferred by some, are 
they always necessary? Perhaps we are overprescribing more costly single-use supplies that contribute to waste in the world. Alternatively, research needs to evaluate a way for these items to be provided in a biodegradable format that is more sustainable.

There are many elements of sustainability that we can mull with respect to people with SCIs and some would argue about the need to discuss this topic. Nevertheless, our clients with SCIs remain the most vulnerable to the impacts of climate change and deserve the most attention. This is a human rights concern. Hence, as we move forward, it is important that we consider the issue of sustainability and SCI and the journal will now add a special section where we will include related submissions. As always, we look for your submissions; and, I am interested to hear your views regarding this topic.

\section{Compliance with ethical standards}

Conflict of interest I am developing a non-profit organization called Telerehabilitation International. I am not receiving any compensation for this work.

Publisher's note Springer Nature remains neutral with regard to jurisdictional claims in published maps and institutional affiliations.

\section{References}

1. Tsai C, Miller A, Huang V, Escalon M, Bryce T. The feasibility and usability of a mobile application for performing home evaluations. Spinal Cord Ser Cases. 2019;5:76.

2. Tyagi N, Amar Goal S, Alexander M. Improving quality of life after spinal cord injury in India using telehealth. Spinal Cord Ser Cases. 2019;5:70. 